\title{
Experimental and theoretical investigations on a CVD grown thin film of polymeric carbon nitride and its structure
}

\author{
Pedro Chamorro-Posada ${ }^{a, *}$, Roberto C. Dante ${ }^{\mathrm{b}}$, José Vázquez-Cabo ${ }^{\mathrm{c}}$, Denisse G. Dante ${ }^{\mathrm{b}}$, \\ Pablo Martín-Ramos ${ }^{\mathrm{d}}$, Óscar Rubiños-López ${ }^{\mathrm{c}}$, Francisco M. Sánchez-Arévalo ${ }^{\mathrm{e}}$ \\ ${ }^{a}$ Dpto. de Teoría de la Señal y Comunicaciones e IT, Universidad de Valladolid, ETSI Telecomunicación, Paseo Belén 15, 47011 Valladolid, Spain \\ ${ }^{\mathrm{b}} R \& D$ Department, 2Dto3D S.r.l.s., Via Revalanca 5, San Firmino, 12036 Revello, Italy \\ ${ }^{\text {c }}$ Dpto. de Teoría de la Señal y Comunicaciones, Universidad de Vigo, ETSE Telecomunicación, Lagoas Marcosende, 36310 Vigo, Spain \\ d EPS, Instituto de Investigación en Ciencias Ambientales (IUCA), Universidad de Zaragoza, Carretera de Cuarte s/n, 22071 Huesca, Spain \\ ${ }^{\mathrm{e}}$ Departamento de reología y mecanica de materiales, Instituto de Investigaciones en Materiales (IIM), Universidad Nacional Autónoma de México (UNAM), Apdo. Postal \\ 70-360, Cd. Universitaria, Mexico City, Mexico
}

\section{A R T I C L E I N F O}

\section{Keywords:}

Carbon nitride

Thin-film

Semi-empirical methods

PM6

Chemical vapor deposition

\begin{abstract}
A B S T R A C T
A polymeric carbon nitride thin film has been grown using chemical vapor deposition. The characterization of the material shows that it has the same molecular composition as a formerly synthesized graphitic carbon nitride powder but both substances differ widely in their structural organization. In particular, our analyses reveal a paradoxical character in which the thin film sample exhibits simultaneously a high degree of organization in the stacking of the polymer sheets with strong inter-layer interactions, as expected from the growth technique, and a complete lack of crystallinity. A comprehensive theoretical study based on massive semi-empirical quantum chemistry computations has permitted to explain the properties of the material and to elucidate fundamental issues regarding the structural conformation of graphitic carbon nitride.
\end{abstract}

\section{Introduction}

Polymeric carbon nitride is a highly versatile material with a broad range of applications including, among others, photocatalysis [1], hydrogen generation [2], photovoltaics [3], and gases [4] and glucose sensing [5]. It was first synthesized by Berzelius and Liebig in 1834 [6]. Pauling and Sturdivant then clarified that this substance contained a $\mathrm{C}_{6} \mathrm{~N}_{7}$ nucleus ("cyameluric nucleus"), which is currently referred to as either heptazine or tri-s-triazine nucleus [7].

There is a widespread agreement on the structure of the material at the one-dimensional and two-dimensional levels, based on a linear polymer with a $\mathrm{C}_{6} \mathrm{~N}_{7}\left(\mathrm{NH}_{2}\right) \mathrm{NH}$ repeating unit. These one-dimensional threads are held together by diffuse hydrogen bonds forming twodimensional sheets [8-11]. The three-dimensional arrangement of the polymer layers is, on the other hand, more controversial. This material generally exhibits a low degree of crystallinity and its morphology can vary widely depending on the synthesis conditions. Notwithstanding, crystalline polymeric carbon nitride has been theoretically predicted [9] using semi-empirical quantum chemistry methods and characterized with XRD and neutron diffraction $[8,12]$, where the combination of these two techniques is imposed by the low degree of crystallinity of the material.

In this work, we address the properties of a thin film of polymeric carbon nitride grown using chemical vapor deposition. This synthesis procedure is expected to provide a good organization of the polymer layers. In fact, the characterization of the material, supported by theoretical investigations, is consistent with a stronger inter-layer interaction than that typically found in powder samples. In spite of this, the material is amorphous.

In order to explain these apparently contradictory properties, we have conducted a comprehensive analysis of the polymeric carbon nitride inter-layer interactions using semi-empirical quantum chemistry methods. This type of computations has played a key role in the understanding of the structure of graphitic carbon nitride in the past $[8,9]$. Semi-empirical neglect of diatomic differential overlap (NDDO) methods can be placed at an intermediate theory level between forcefield and ab-initio calculations. They are based on a series of approximations, and the data is obtained from given training sets. The most recent PM6 and PM7 parametrizations provide a performance comparable to density functional theory and ab-initio methods in the

\footnotetext{
* Corresponding author.

E-mail address: pedcha@tel.uva.es (P. Chamorro-Posada).
} 
prediction of ground state geometries, while their largely reduced computational cost permits to address very large molecular systems and solid-state materials [13], especially with the advantage provided by the parallelization of some parts of the code [14]. In this work, we take advantage of the computational efficiency of this method to perform a broad study of the inter-layer interaction properties of polymeric carbon nitride.

We first present the results of the full characterization of the carbon nitride thin-film. The paradoxical properties of this material are then explained on the basis of an extensive theoretical study.

\section{Experimental}

\subsection{Materials and synthesis procedure}

The carbon nitride film was synthesized by thermal chemical vapor deposition (CVD) at the temperature of $600{ }^{\circ} \mathrm{C}$ in air atmosphere. The precursor, cyanurate of melamine supplied by Nachmann S.r.l. with purity $>99 \%$, was put on the bottom of the ceramic crucible and a ceramic glass square of $5 \times 5 \mathrm{~cm}$ was placed on the top, closing the crucible. Thus, the gaseous molecules were deposited on the glass surface facing the inner walls of the crucible. The film grew on the surface of the ceramic glass, and was extracted from the muffle after $30 \mathrm{~min}$ of treatment.

\subsection{Characterization and computation}

The characteristics of the synthesized thin film were evaluated by Xray powder diffraction, using a Bruker (Billerica, MA, USA) D8 Discover diffractometer $(\mathrm{CuK} \alpha=1.5418 \AA$ ); and by attenuated total reflectance Fourier-transform infrared spectroscopy (ATR-FTIR) with an Agilent (Santa Clara, CA, USA) Cary 630 spectrophotometer.

UV-Vis spectra of the powder and thin film samples were obtained with an Agilent UV-Vis Cary 100 in reflection mode.

The Raman spectra were obtained with a Micro-HR-Raman Horiba Jobin Yvon LAbRam-HR800 with confocal microscope using a $\times 10$ objective and $532 \mathrm{~nm}$ Raman excitation wavelength.

The thin film was characterized both using contact and interferometry profilometry techniques. Stylus profilometry measurements were performed using a Bruker a Dektak XT high resolution profiler. A Wyko NT1100 optical system was used for non-contact 3D surface measurement.

For a more detailed characterization report of powder polymeric carbon nitride, the interested reader is referred to previous works [15-17].

The sample surface was also investigated using scanning electron microscopy (SEM) with a JEOL (Peabody, MA, USA) JSM6700F apparatus at $5 \mathrm{kV}$.

A lamella of the sample was prepared with the lift out technique using a FEI Helios Nanolab 600 , working with a Ga ${ }^{+}$ion beam at $30 \mathrm{kV}$. Its morphology was studied investigated by transmission electron microscopy (TEM) with a JEOL JEM2010F apparatus at $200 \mathrm{kV}$, equipped with an energy dispersion spectroscopy (EDS) probe.

The semi-empirical quantum chemistry computations were based on the PM6 [18] Hamiltonian implemented in the MOPAC2016 software package using acceleration in multithreaded shared memory architectures [14]. The numerical calculations were performed in a server with a 12 threads Intel Xeon E5-1650v2 6 Core 3,5GHz processor and a Linux operating system. The electronic spectrum, for the PM6 optimized geometry, was calculated via the intermediate neglect of differential overlap/spectroscopic (INDO/S) method and configuration interaction with singles (CIS) $[19,20]$ using the ORCA electronic structure package version 2.9.1 [21].

The chemical composition of the sample was examined ex situ by XPS surface measurements. The $\mathrm{C} 1 \mathrm{~s}, \mathrm{O} 1 \mathrm{~s}, \mathrm{~N} 1 \mathrm{~s}$ and survey spectra were recorded using a Thermo Scientific (Waltham, MA, USA) K-Alpha

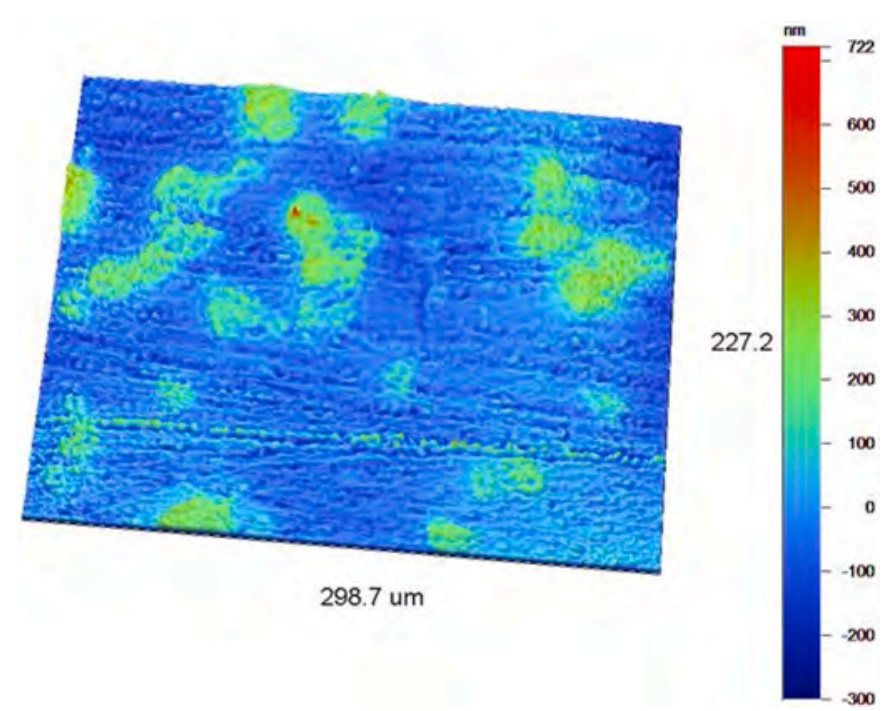

Fig. 1. Surface topography in a region of the sample measured using WLOI in the Vertical Scanning Interferometry mode.

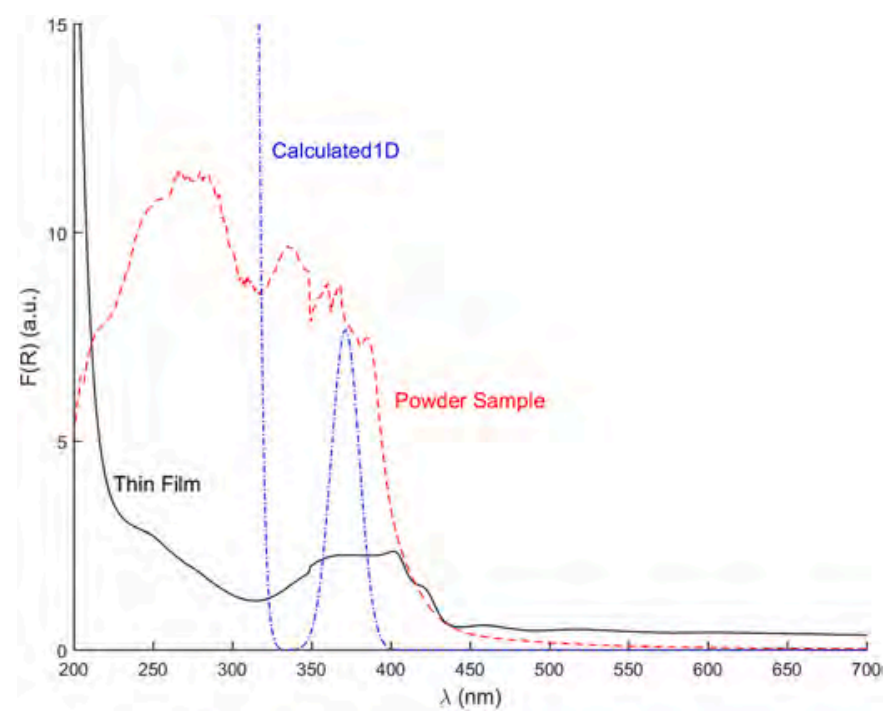

Fig. 2. UV-Vis spectra of the thin film and powder samples. The $F(R)$ parameters obtained from the Kubelka-Munk model applied to the diffuse reflectance measurements are shown. The spectrum of a fourth-order melon oligomer calculated using the INDO/S method is also shown for comparison purposes.

instrument. Monochromatic X-ray source $\mathrm{Al} \mathrm{Ka}(1486.6 \mathrm{eV})$ was used for experiments. Spectra were acquired at 10-9 mbar. The X-ray monochromatic spot is $400 \mu \mathrm{m}$ in diameter. Residual vacuum in the analysis chamber was maintained at around $6 \times 10^{-9}$ mbar. The binding energies (BEs) positions on unsputtered surfaces were calibrated by setting the $\mathrm{C} 1 \mathrm{~s}$ photopeak corresponding to aliphatic carbon at $285.0 \mathrm{eV}$.

The atomic concentrations were determined from the XPS peak areas using the Shirley background subtraction technique and the Scofield sensitivity factors. A wide scan survey spectrum were used to identify and quantify the elements in the sample. High resolution narrow scans were used to build the chemical state assessment table, which is presented in full detail. 


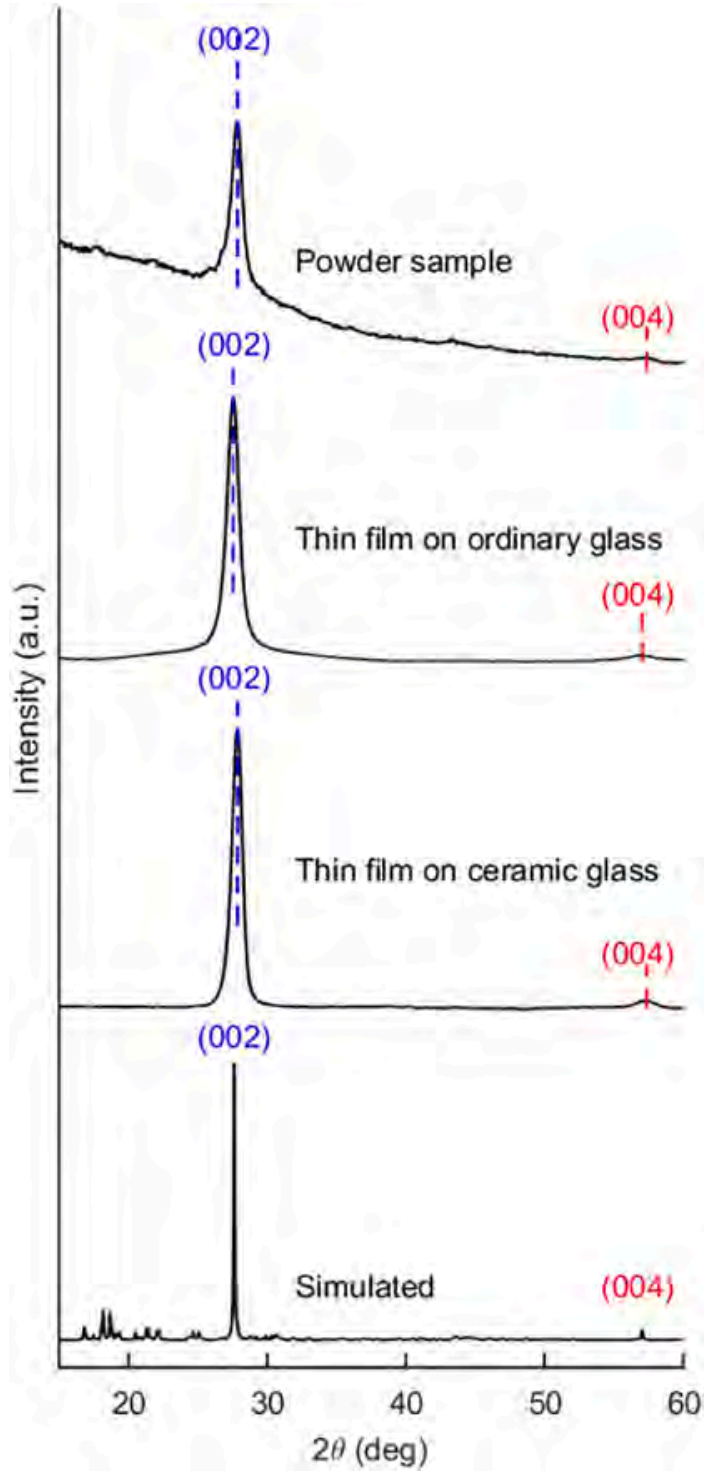

Fig. 3. Diffractograms obtained from a powder sample, a thin film grown on ordinary glass, a thin film on ceramic glass and the simulated diffractogram for the 3D crystal geometry theoretically predicted in [9]. From top to bottom, the positions of the (002) peaks are: $27.86^{\circ}, 27.55^{\circ}, 27.86^{\circ}$, and $27.62^{\circ}$, the (004) are placed at: $57.40^{\circ}, 57.05^{\circ}, 57.38^{\circ}$, and $57.04^{\circ}$.

\section{Results}

\subsection{Characterization}

The film thickness and the surface topography of the thin film sample were characterized using both high resolution stylus profilometry and while light optical interferometry (WLOI). The thickness of the sample grows in a short distance from the outer rim to a value of $2 \mu \mathrm{m}$ according to our measurements. The surface displays a series of islands of variable height ranging between less than $100 \mathrm{~nm}$ to a few hundreds of nanometers, as illustrated in Fig. 1. In the analyzed area, 17\% of the total surface is occupied by these islands. The values of the 3D roughness parameters measured with WLOI yield $\mathrm{Sa}=66.29 \mathrm{~nm} \mathrm{Sq}=89.44 \mathrm{~nm}$ for the average and RMS roughnesses, respectively, with skewness Ssk = 1.20 and Kurtosis $=4.97$.

Fig. 2 displays the Kubelka-Munk function $F(R)=(1-R)^{2} /(2 R)$, where $R$ is the sample reflectance, obtained from the diffuse reflectance of both the thin film and the powder sample. The Tauc fits give measured

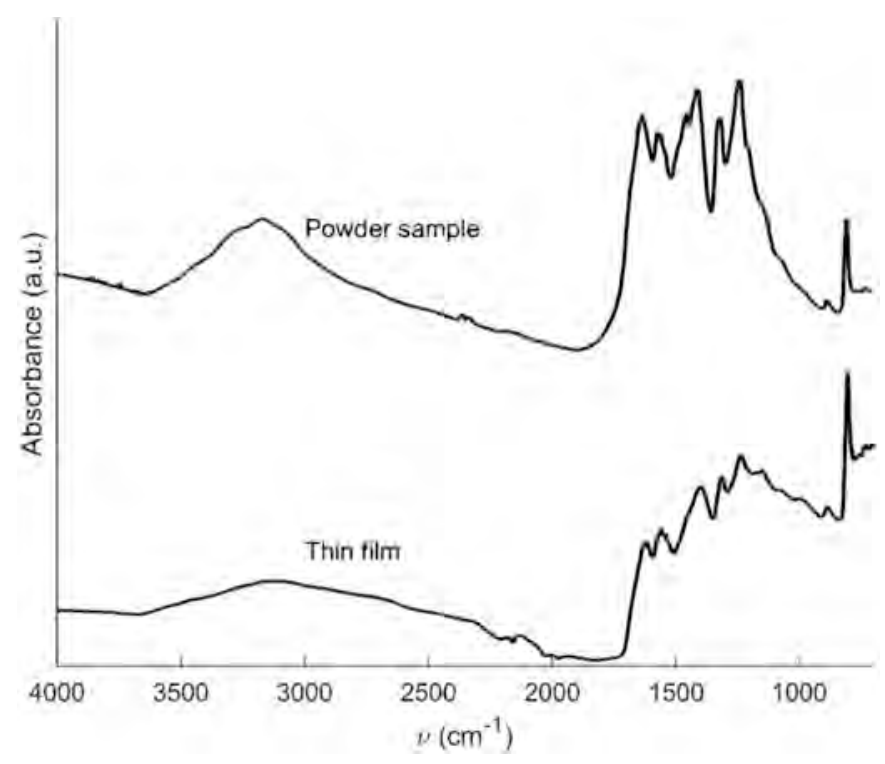

Fig. 4. Measured IR spectra of the thin film (bottom) and the reference powder sample (top).

band gaps of $2.9 \mathrm{eV}$ and $3.1 \mathrm{eV}$ for the thin film and powder samples, respectively. The different shapes for the film and powder spectra can be attributed to the broadening of the linewidth of the electronic transitions in the bulk material. The small oscillations in the thin film sample spectrum are due to the interference produced by multiple reflections in the film interfaces. For comparison purposes, the absorption spectrum of the fourth order 1D melon oligomer, with eight heptazine units, is also shown. The calculation of varying order oligomers shows has little impact in the calculated spectra of melon [9]. This can be attributed to the localization of the excitations within the heptazine unit, in contrast with the behavior of delocalized excitations in conventional semiconductors [9].

Fig. 3 compares the diffractograms obtained from thin film and powder samples and those simulated using the 3D crystal geometry predicted in [9]. The measurements of the thin film samples have been performed with the X-ray beam impinging on the film plane. The peaks arising from the presence of virgilite in the ceramic glass have been eliminated from the diffractogram. Experimental and theoretical studies on the crystal structure of polymeric carbon nitride $[9,12]$ show two layers with an A-B stacking motif in the crystal unit cell. Therefore, the main reflection corresponds to the (002) plane. Even though no true 3D crystal ordering is present in the CVD thin-film samples, for the sake of clarity, we will keep this notation for the reflections at the, otherwise, well-formed layers in these films. All the traces in Fig. 3 display a main peak due to the (002) interlayer reflections. The signal corresponding to the second order reflection at the (004) plane is also present with a much lesser intensity. It is noteworthy that both thin-film samples display only these features, which are related to the layer planes, whereas additional (fainter) peaks that would be associated to the formation of proper crystals [12], such as those shown in the simulated diffractogram, are totally absent. On the other hand, these additional features, although dimmed by the noisy background due to the low crystallinity of the material, are visible in the diffractogram of the powder sample, which indicates the existence of micro-crystals within a mostly amorphous material.

The analysis of the positions and widths of the peaks in a X-ray powder diffractogram are very useful to determine the stacking order of layered materials [25-27]. The position of the main (002) peak coincides in the thin-film over ceramic glass and powder samples and it is slightly shifted in the thin film deposited on ordinary glass. The widths, measured as the full-width at half the maximum intensity (FWHM), of 


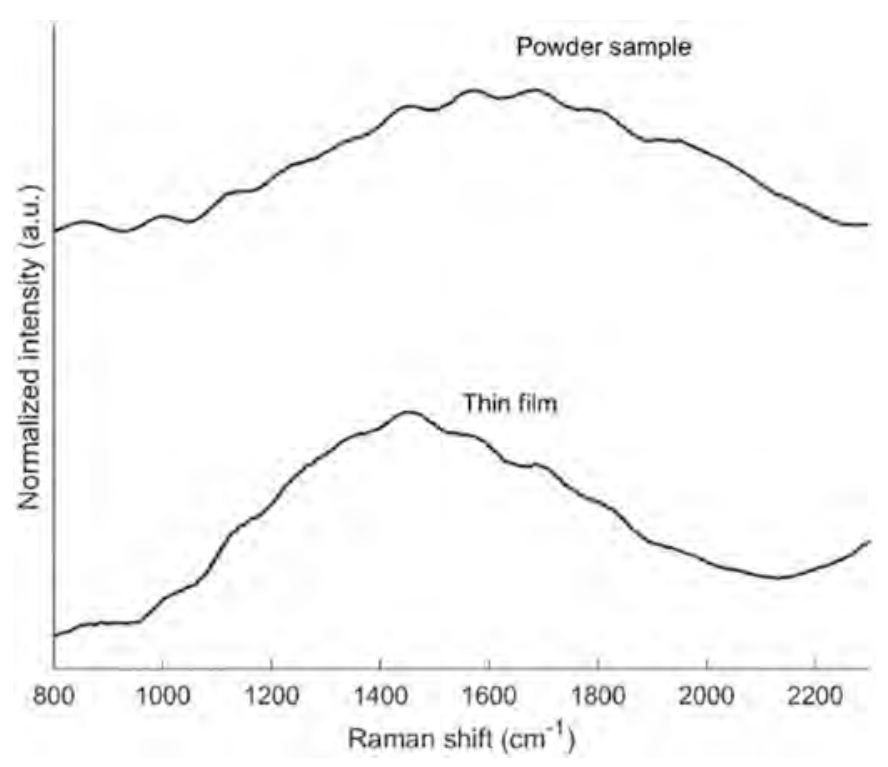

Fig. 5. Raman spectrum of the thin film (bottom) and powder (top) sample measured with excitation at $532 \mathrm{~nm}$.

the (002) peaks of the CVD thin films deposited on ordinary and ceramic glasses are $1.11^{\circ}$ and $0.89^{\circ}$, respectively, with a significant increase of the width of the peak of the sample deposited on ordinary glass as compared to that of ceramic glass. These two alterations of the characteristics of the (002) peak of the thin-film samples can be explained as the result of an increase of the average interlayer separation and of the

(a)

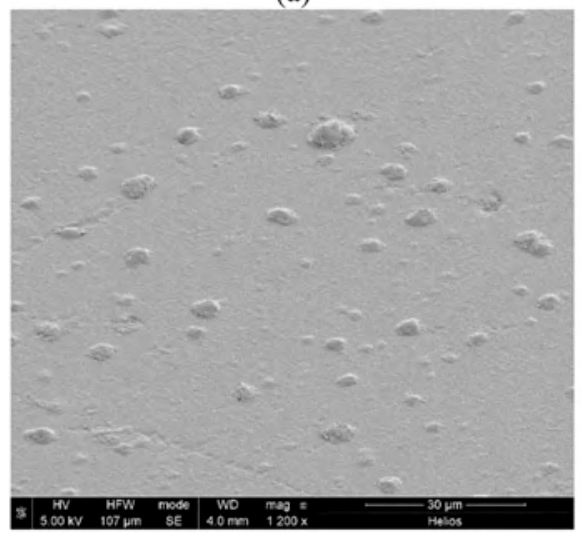

(c)

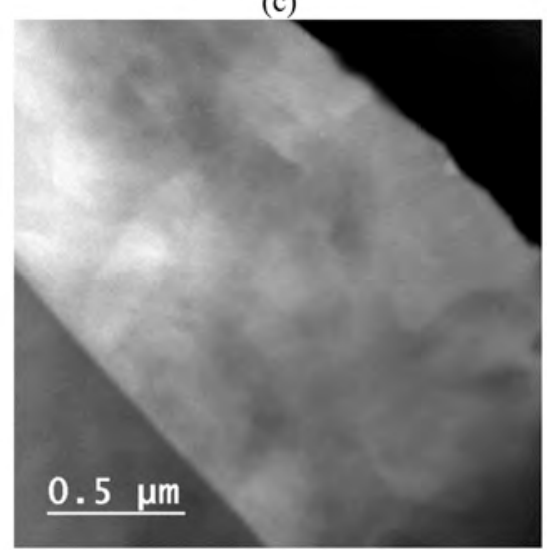

dispersion in the values of the interlayer spacing, respectively, in the ordinary glass sample. These effects can be attributed to a deformation of the glass substrate at the CVD temperature that decreases the level of organization in the stacking of the $2 \mathrm{D}$ polymer.

Fig. 4 shows the FTIR spectra of the powder and thin film samples. The absorption bands in the IR spectrum of polymeric carbon nitride have been analyzed in detail in [11]. The information provided by the IR spectra is twofold: Firstly, one can observe the positions of the bands corresponding to the vibration modes, and, secondly, the relative intensities of the various observed bands. As regards the positions of the bands, they are exactly the same in both cases, powder and thin layer, in all the spectral range and this means that the two materials are essentially the same. As to the relative intensities, and taking into consideration the shifts of the baselines, the only remarkable difference is the relative intensity of the peak at $814 \mathrm{~cm}^{-1}$ with respect to all the others. This relative difference can be interpreted as the result of the fact that the deformation (backbone) mode of the polymer at $814 \mathrm{~cm}^{-1}$ [11] is less restrained in comparison with all the remaining localized vibration modes in the thin layer than in the powder material. These long-range backbone vibrations occur most often in the far infrared and terahertz domains and, as it happens in our case, their spectral responses provide direct information about the structural organization of materials, in particular of those with a layered conformation (see [13] and references therein).

Fig. 5 displays the Raman spectra, obtained with excitation at 532 $\mathrm{nm}$, of the powder and thin film samples. The measured spectra display a band near the $\mathrm{G}$ region characteristic of $s p^{2}$ carbon materials, which lies around $1560 \mathrm{~cm}^{-1}$ in graphite. This is expected since $\mathrm{C}-\mathrm{C}$ and $\mathrm{C}-\mathrm{N}$ modes have similar vibrational frequencies [22]. This band is superimposed to a wavy background due to the response from the glass that supports the thin film and that of the holder used in the characterization

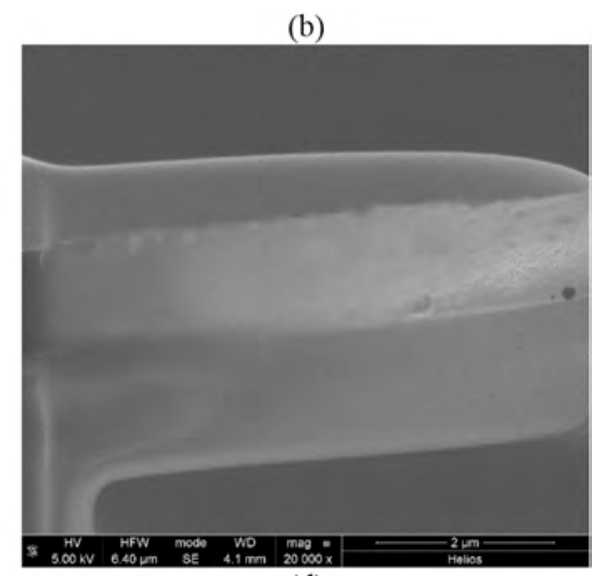

(d)

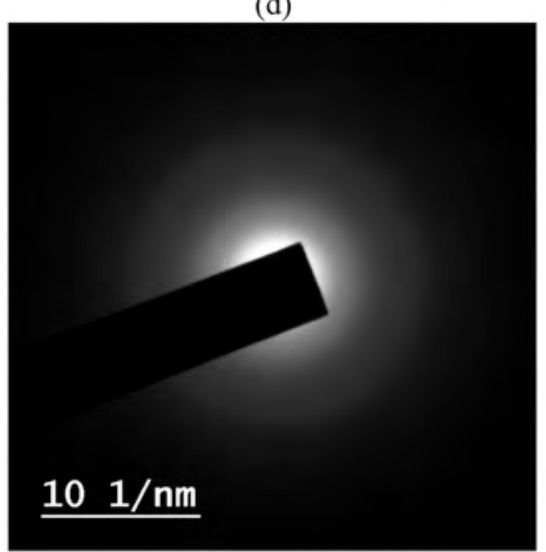

Fig. 6. a Panels (a) and (b) display SEM micrographs of the surface of the sample and a lamella obtained using FIB, respectively. (c) TEM image of a lamella and (d) SAED pattern. 


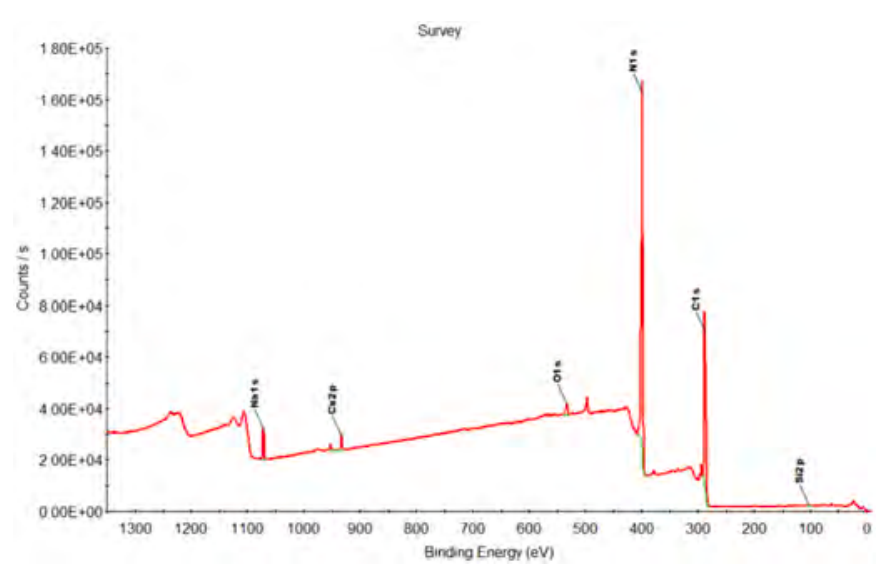

Fig. 7. Survey of the carbon nitride thin film on ceramic glass.

Table 1

Data from survey spectrum with atomic percentage of detected elements.

\begin{tabular}{lllll}
\hline Name & Peak BE & FWHM eV & Area (P) CPS eV & at.\% \\
\hline C1s & 287,65 & 2,47 & $254,630,93$ & 49,13 \\
N1s & 399,16 & 2,89 & $416,624,65$ & 47,36 \\
O1s & 532,59 & 4,82 & $21,033,45$ & 1,59 \\
Na1s & 1071,88 & 2,45 & $31,462,53$ & 1,35 \\
Si2p & 102,59 & 2,26 & 1335,74 & 0,29 \\
Cu2p & 933,02 & 2,49 & $23,481,38$ & 0,28 \\
\hline
\end{tabular}

of the powder sample. The differences between the two spectra are consistent with a relative shift of the position in the $\mathrm{G}$ peak, as described in Ref. 22, due to the increase of topological disorder.

The results of TEM and SEM analysis of the thin film are shown in Fig. 6. Panels (a) and (b) show, respectively, a SEM micrograph of the film surface, displaying the islands that were also found in the profilometric study, and a lamella obtained using FIB. Panels (c) and (d) display a TEM image of the lamella and the selected area diffraction (SAED) analysis, evidencing the amorphous character of the sample.

The survey spectrum of XPS is shown in Fig. 7 and Table 1 reports the atomic concentration of the detected elements. It is notable that the ratio of $\mathrm{N}$ to $\mathrm{C}$ is 0.96 instead of 1.33 , as found in the polymeric carbon nitride powder [15]. As opposed to other characterization methods, XPS is a surface-sensitive technique. The optical absorption measured in a FTIR spectrometer, on the other hand, provides information accumulated over the light passage across the whole sample, with a negligible contribution from any anomaly localized at its surface. As discussed above, the observation of exactly the same bands in the IR spectra evidences that both samples, powder and thin film, share the same molecular structure and the differences between the XPS results have to be due to an interfacial effect in the thin film sample. We attribute the discrepancy in the XPS measurements to the existence of the islands spread over the surface of the thin film that could be remains from the CVD process.

A further scrutiny of the deconvolution of the C1s and N1s shown in Fig. 8 and Table 2 reinforces this interpretation of the results obtained from the characterization of the thin film sample. As regards N1s, the $\mathrm{N} 1 \mathrm{sp}^{3}$ species can be attributed to either $\mathrm{NH}$ bridges or $\mathrm{NH}_{2}$ terminal groups, while the $\mathrm{N} 1 \mathrm{sp}^{2}$ signal corresponds to nitrogen atoms within the heptazine units. Respecting C1s, the peak identified as $\mathrm{C} 1 \mathrm{~s} \mathrm{sp}^{2}-\mathrm{Nsp}^{2}$ in Fig. 8 corresponds to the carbon inside the heptazine rings bonded to nitrogen atoms of the ring with the same hybridization, and $\mathrm{C} 1 \mathrm{~s} \mathrm{sp}^{2}$ $\mathrm{Nsp}^{3}$ corresponds to those carbon atoms of the heptazine units bonded to nitrogens with $\mathrm{sp}^{3}$ hybridization such as amine groups such the terminal $\mathrm{NH}_{2}$, and $\mathrm{NH}$ which is the bridge between heptazine units. We attribute $\mathrm{C} \mathrm{sp}^{3}$, around $20 \%$ of the total, to other types of carbon originated as remains from the CVD precursors and deposited at the islands on top of the carbon nitride film. This contribution altered the results of the XPS scan with an overestimation of $\mathrm{C} 1 \mathrm{~s}$ due to the spurious $\mathrm{C} 1 \mathrm{sp}^{3}$, and its cancellation from the analysis allows the reconciliation of the results obtained from the XPS analyses of powder and thin film samples.

\subsection{Theoretical}

As commented above, there is a well-stablished consensus about the structural properties of the 2D layers [8,9] that are the building blocks of different polymeric carbon nitride conformations. As regards the $3 \mathrm{D}$ frameworks, the situation is far more convoluted. This material can exhibit a wide variety of shapes, like crumpled nanosheets or particles with different shapes. The existence of $3 \mathrm{D}$ crystals is theoretically possible [9] and has been experimentally confirmed [12], but the typical sizes of these crystals are very small and the material generally exhibits a predominantly amorphous structure. In this work, we have studied a thin film sample for which the analyses performed indicate a wellorganized 3D stacking of the polymer layers and yet, at the same time, a totally amorphous character.

Table 2

Peak positions and atomic percent of C1s and N1s species.

\begin{tabular}{|c|c|c|c|c|}
\hline Name & Peak BE & FWHM eV & Area (P) CPS eV & at.\% \\
\hline $\mathrm{N} 1 \mathrm{~s} N$-sp ${ }^{2}$-ring & 399,08 & 1,24 & $25,880,5$ & 56,52 \\
\hline $\mathrm{N} 1 \mathrm{~s} \mathrm{~N}-\mathrm{sp}^{3}$ & 400,39 & 2,67 & $19,692,12$ & 43,03 \\
\hline N1s N-Ox & 404,89 & 0,85 & 204,88 & 0,45 \\
\hline Total & & & & 100,00 \\
\hline $\mathrm{C} 1 \mathrm{~s} \mathrm{sp}^{3}$ & 285 & 1,4 & 6412,01 & 20,25 \\
\hline $\mathrm{C} 1 \mathrm{~s} \mathrm{sp}^{2} \mathrm{~N} \mathrm{sp}^{3}$ & 287,1 & 2,22 & $13,607,94$ & 43,02 \\
\hline $\mathrm{C} 1 \mathrm{~s} \mathrm{sp}^{2} \mathrm{~N}-\mathrm{sp}^{2}$ & 288,71 & 1,13 & $11,612,69$ & 36,74 \\
\hline Total & & & & 100,00 \\
\hline
\end{tabular}

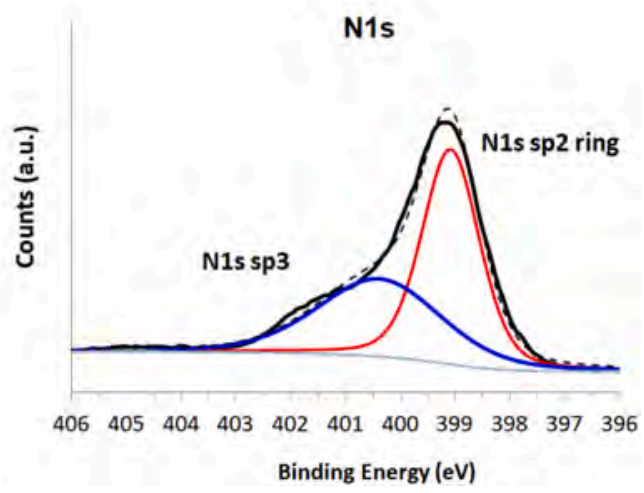

Fig. 8. Peaks of $\mathrm{C} 1 \mathrm{~s}$ and N1s with deconvolutions curves and their attribution. 


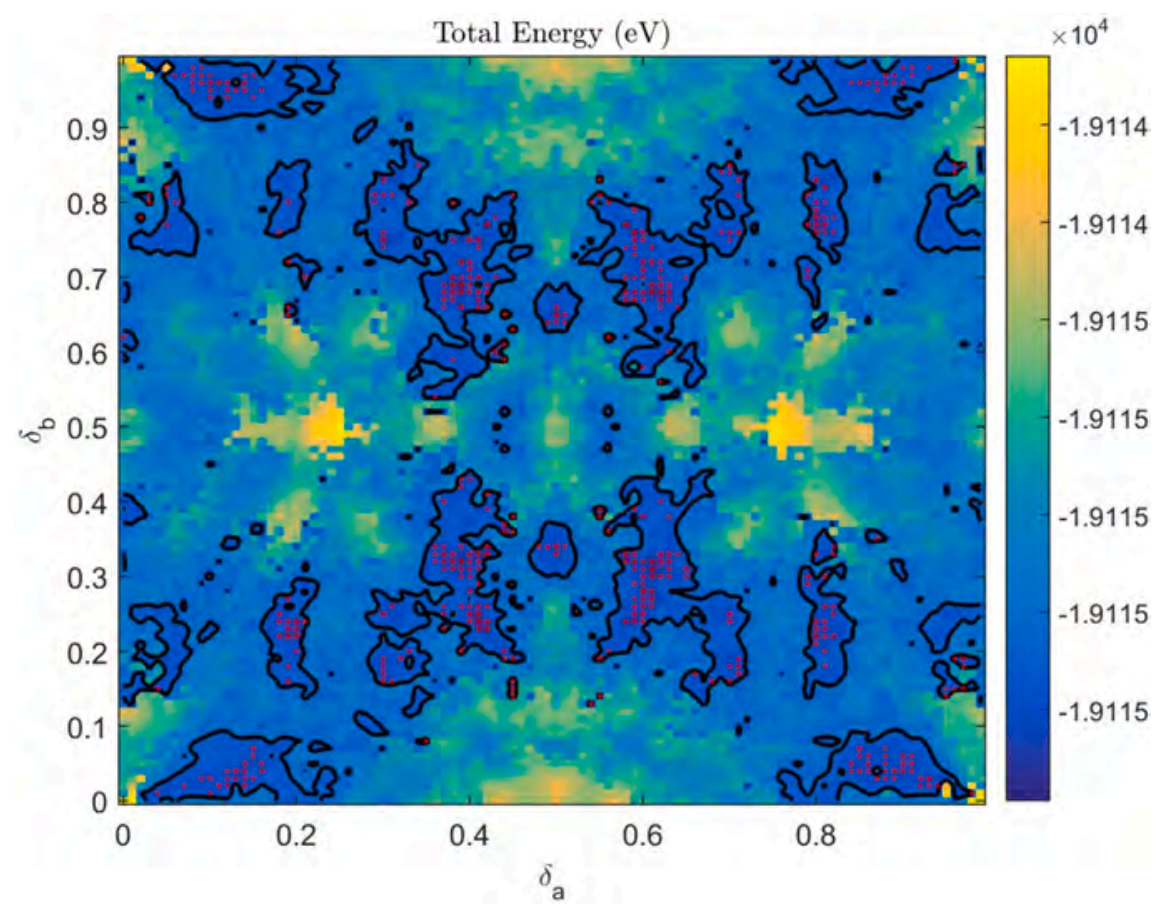

Fig. 9. Computed total energies (in eV) for the space of 10,000 different initial two-layer configurations studied. The black contour bounds the initial configurations leading to total energies within the lower $20 \%$ percentile of the energy distribution. Red dots mark the lowest energy geometries. (For interpretation of the references to color in this figure legend, the reader is referred to the web version of this article.)

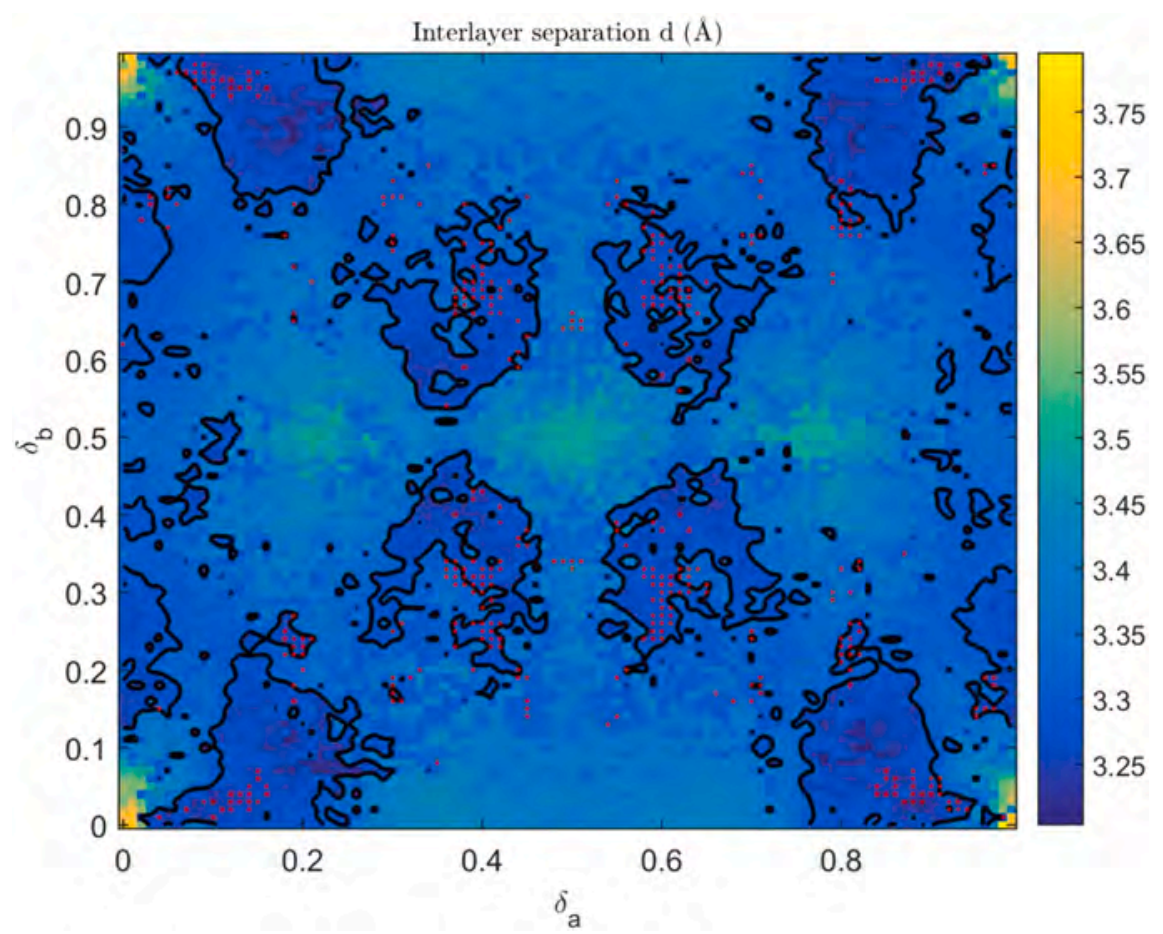

Fig. 10. Interlayer separation of the optimized geometries (in $\AA$ ). The black contour encloses initial configurations leading to interlayer separations below $3.35 \AA$. Red dots mark the lowest energy geometries. (For interpretation of the references to color in this figure legend, the reader is referred to the web version of this article.)

In order to gain a clear understanding of the structural properties of the material, we have carried out a massive computational study of the interlayer interactions. Starting from the 2D periodic optimized geometry of [9], a second layer was placed with a separation corresponding to that of the 3D crystal geometry of [9]. A relative in-plane shift was applied to this second layer. The shift was determined by the translation vector $\mathbf{t}=\delta_{\mathrm{a}} \mathbf{a}+\delta_{\mathrm{b}} \mathbf{b}$, where $\mathbf{a}$ and $\mathbf{b}$ are the $2 \mathrm{D}$ lattice vectors and $\delta_{\mathrm{a}}$ and $\delta_{\mathrm{b}}$ systematically take values in the interval $[0,1]$. Using this scheme, we have generated a set of initial solutions creating a grid of 10,000 configurations regularly sampling the space of all possible two-layer 

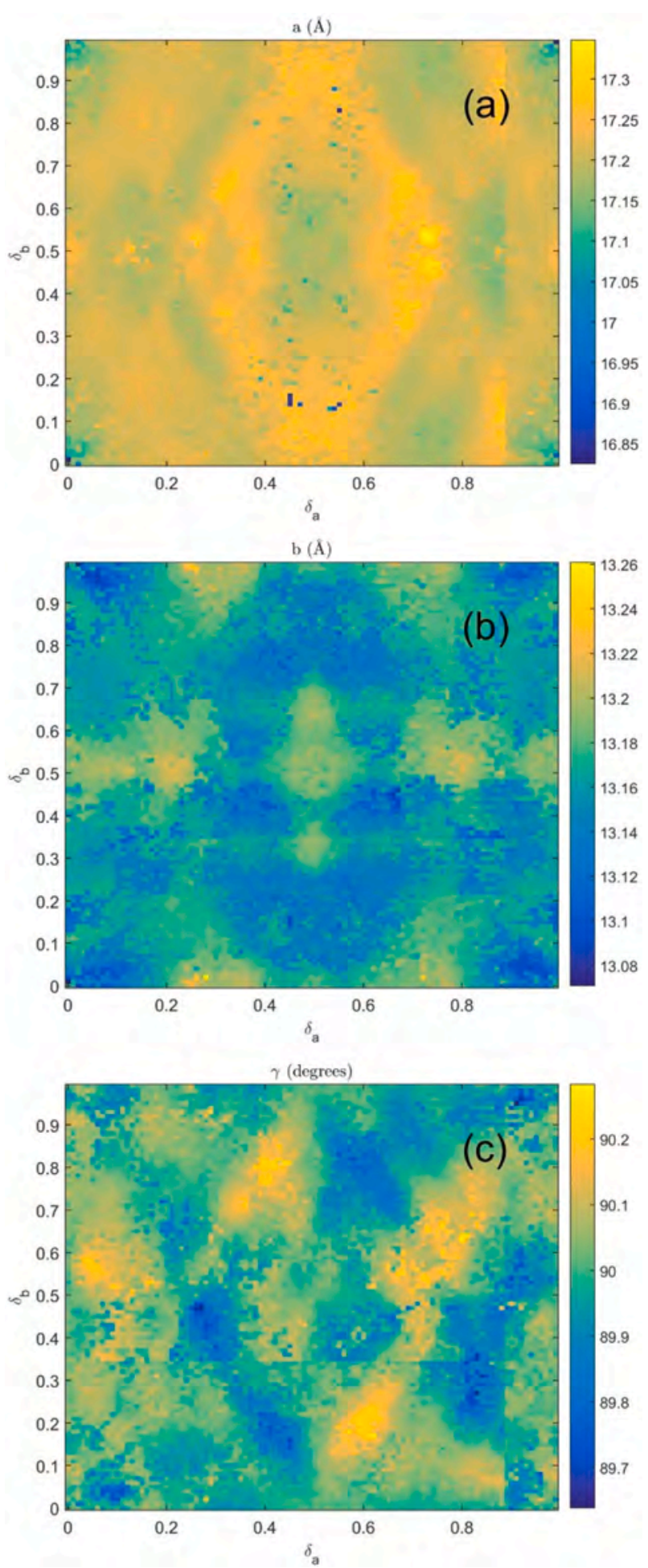

Fig. 11. Lattice parameters (a) $a$ (b) $b$ and (c) $\gamma$ of the 2D periodic optimized two-layer geometries as a function of the initial geometry configuration parameters $\delta_{\mathrm{a}}$ and $\delta_{\mathrm{b}}$.

conformations. These geometries have been optimized as 2D periodic structures using the PM6 Hamiltonian.

The final values of total energy obtained from the geometry optimizations in the $\left(\delta_{\mathrm{a}}, \delta_{\mathrm{b}}\right)$ grid are displayed in Fig. 9. The results show that instead of a localized deep minimum that would favor a definite arrangement of the two-layer system, and the subsequent formation of a single crystal structure upon layer piling, there exist extensive broad

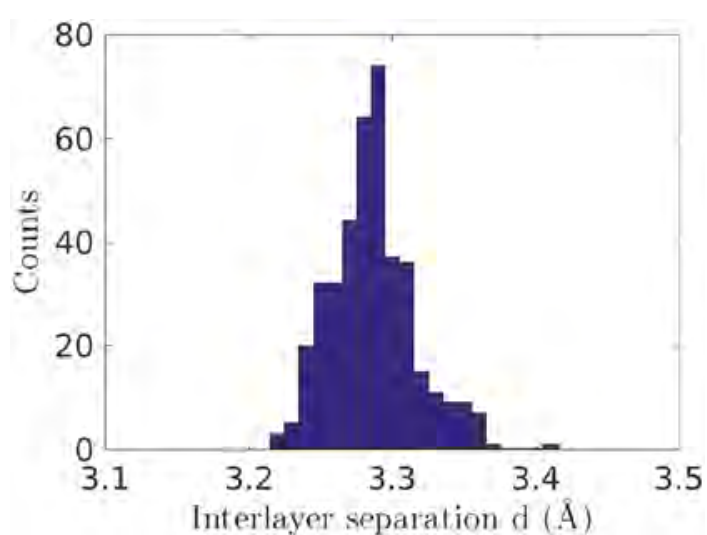

Fig. 12. Histogram of the interlayer separation of the subset of lowest energy sites in the $\left(\delta_{\mathrm{a}}, \delta_{\mathrm{b}}\right)$ parameter space marked with red dots in Figs. 8 and 9.

valleys of low total energy values. These act as multiple manifolds of feasible arrangements of the two-layer system. The black contour in the figure outlines the region within the lower $20 \%$ percentile of the total energy.

Fig. 10 depicts the interlayer separation for each optimized structure in the $\left(\delta_{\mathrm{a}}, \delta_{\mathrm{b}}\right)$ plane. In order to estimate this value, the coordinates along the direction perpendicular to the plane defined by the $\mathbf{a}$ and $\mathbf{b} 2 \mathrm{D}$ lattice vectors are computed, at each layer, for the heavier atoms ( $\mathrm{C}$ and $\mathrm{N}$ ) in one unit cell. The estimated value of the interlayer separation is the difference between the average values of these two sets of coordinates. It is interesting to recall that the heptazine units are positioned with small relative tilts within the polymer plane [9]. The comparison of Figs. 9 and 10 shows that there is a good correlation between the lowest energy sites and those with low interlayer separations, even though it is noteworthy that the shortest separations do not coincide with the lowest formation energies.

Another consideration of relevance for the properties of the layer stacking and the final material conformation is the matching of the lattice parameters. The existence of large variations in the lattice constants over the configuration space of two-layer systems would provide a selection mechanism by which only lattice matched layer combinations would contribute to a final 3D arrangement. Fig. 11(a), (b) and (c) depicts, respectively, the lattice parameters $a, b$ and $\gamma$. These plots show that the dispersion of the lattice parameters is very small over the whole $\left(\delta_{\mathrm{a}}, \delta_{\mathrm{b}}\right)$ plane. Therefore, lattice matching is not a concern in the process of structural organization of the material.

Next, we conducted a more detailed analysis based on a restricted set comprising the most energetically favorable solutions. We selected the 400 lowest energy configurations, that corresponded to a $4 \%$ of the total number of configurations studied. These values are marked as red dots in Figs. 9 and 10. In Fig. 9, it can be appreciated that this subset is evenly distributed over the low energy regions comprising advantageous geometries. At the same time, this subset provides an extensive sampling of these regions. Fig. 12 shows the histogram of the distribution of interlayer separations for this subset. This distribution is consistent with the PXRD measurements of the thin film, shown in Fig. 3.

This massive computational study using quantum chemistry methods permits to explain the contradictory structural properties of the CVD thin film sample. During the growth process, the layers are deposited in a very orderly manner, creating a structure with a strong interlayer interaction. At the same time, there are not preferred arrangements for the layers added to the stack that would result in A-A-A-..., A-B-A-B..., or another similar periodic pattern but, instead, a multiple manifold of comparable energy configurations is found. This produces a material with a highly ordered stacking of layers but, at the same time, amorphous. This analysis also provides an explanation for the difficulty of creating large crystals and the fact that the samples synthesized in 


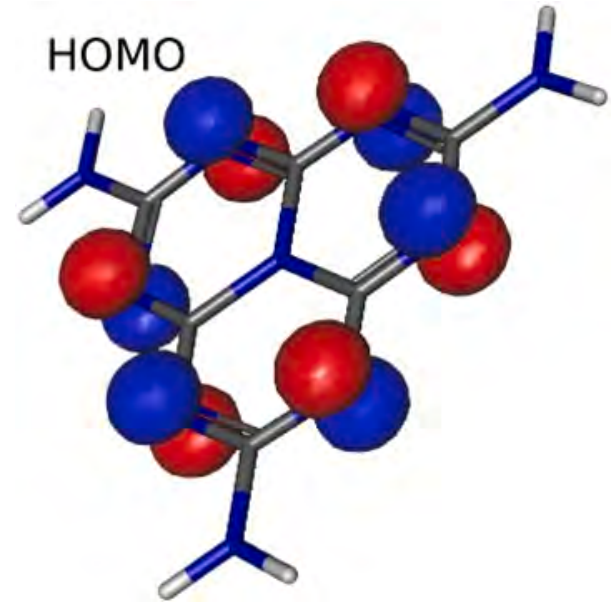

Fig. 13. Highest occupied molecular orbital of the melon monomer.

different manners can display different morphologies, in general, with a very low degree of crystallinity.

We now address the most important question of why the energy distribution among configurations in $\left(\delta_{\mathrm{a}}, \delta_{\mathrm{b}}\right)$ space has the particular shape depicted in Fig. 9. As discussed in the XPS section, one can distinguish between different nitrogen sites in the carbon nitride polymer structure according to their electronic properties: nitrogens at the terminal amino groups, at the secondary amino linkages, at the centers of the heptazine rings and those at the peripheral positions in the heptazine units. These peripheral nitrogen sites, subject of $\mathrm{sp}^{2}$ hybridization, are engaged in two $\sigma$ bonds with an electron pair occupying an inplane non-bonding orbital and an electron contributing to the $\pi$-system. The heptazine core of the melem unit is a highly stable anti-aromatic $\pi$-system [23]. This anti-aromatic character is confirmed by the electronic density distribution in the highest occupied molecular orbital of the melem monomer displayed in Fig. 13 that corresponds to an antibonding $\pi$ orbital with the electronic density localized at the nitrogen atoms placed over the exterior contour of the three fused triazine rings. As discussed below, quantum chemistry computations indicate that these nitrogen sites can play a role as binding centers for 3D carbon nitride arrangements distinct to that of the extended $\pi$-system.

The melon chains in the $2 \mathrm{D}$ polymer layers are kept together by a network of $\mathrm{N}-\mathrm{H} \cdots \mathrm{N}$ bonds with the peripheral nitrogen atoms. As regards the stacking of these layers, the Van der Waals interactions between planar anti-aromatic $\pi$ systems are far less understood than their aromatic counterparts [24]. The crystal structure of the melem monomer indicates that the interaction mechanism between the planar units in the arrangement of the 3D molecular crystal is also dominated by hydrogen bonds with the participation of the peripheral nitrogen atoms [28,29]. Nevertheless, the substitution of the amino groups impedes the formation of hydrogen bonds and results in a crystal structure where the melem platelets are kept together by $n-\pi$ interactions between a peripheral nitrogen atoms and the heptazine cores of nearby molecules [30]. This results in a separation between layers of $3.45 \AA$ [30], slightly larger than the interlayer distance measured for carbon nitride (3.2 $⿱$ ) $)$.

Therefore, there exist different mechanisms that can be responsible for the binding of the carbon nitride layers. In this case, the interaction takes place between well-formed planar structures, which hinders the contribution from $n-\pi$ interactions or hydrogen bonds. Instead, two types of possible interaction mechanisms between the melon planar structures: interplanar interactions between peripheral nitrogen atoms and between the $\pi$-systems of face-to-face heptazine cores are found. The extensive computational survey performed in this work has permitted to evaluate the relative relevance of these mechanisms in the formation of the 3D solid according to the total formation energies and the resulting inter-planar distances by a detailed inspection of the optimized

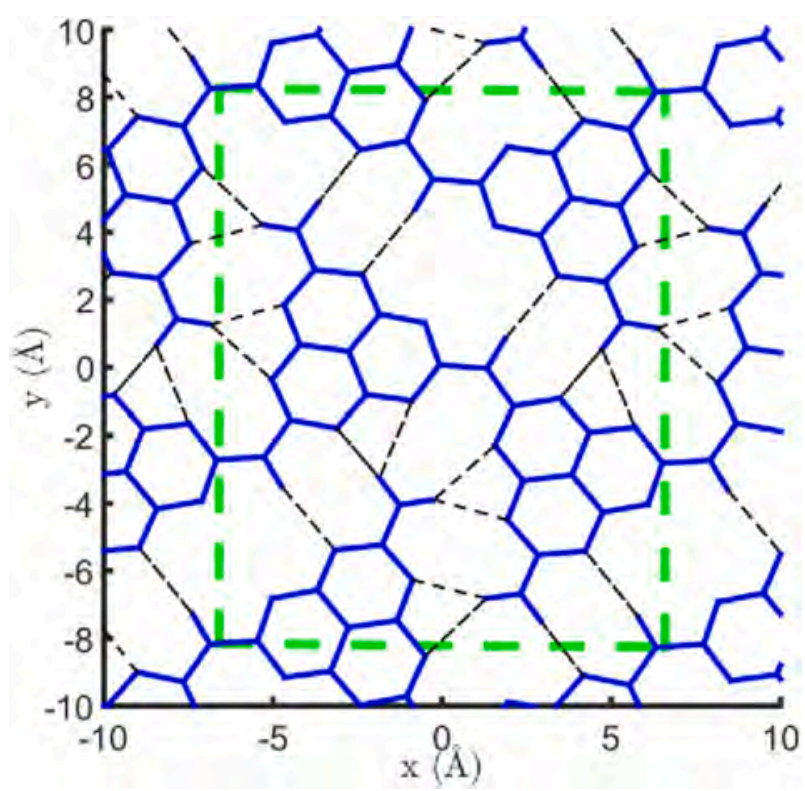

Fig. 14. The network of $\mathrm{N}-\mathrm{H} \cdots \mathrm{N}$ bonds that keep together polymer sheet are marked using dashed black lines. The geometry has been calculated using the PM6 Hamiltonian [9]. The unit cell boundary, with the $a$ axis directed along the $y$ direction, is outlined with a green dashed line. The distances between the $\mathrm{H}$ and the peripheral $\mathrm{N}$ atoms in the hydrogen bonds range between $1.95 \AA$ and $2.51 \AA$. (For interpretation of the references to color in this figure legend, the reader is referred to the web version of this article.)

geometries. The investigations presented herein show that interactions between peripheral nitrogen atoms are the prevalent interaction mechanism between carbon nitride sheets in the most energetically favorable configurations.

We first evaluate the importance of the $\mathrm{N}-\mathrm{H} \cdots \mathrm{N}$ bonds in the interlayer interactions. The $\mathrm{H} \cdots \mathrm{N}$ distances in the $2 \mathrm{D}$ linkage network shown in Fig. 14 range between $1.95 \AA$ and $2.51 \AA$. When the distances for possible inter-layer $\mathrm{H} \cdots \mathrm{N}$ bonds are evaluated in the optimized twolayer systems, the values are always larger than $2.79 \AA$. This observation permits to dismiss the role of, otherwise dominant, hydrogen bonds as a relevant interaction mechanism in the stacking of carbon nitride layers.

Fig. 15 displays the geometries of the four most energetically favorable optimized configurations. In this figure, zenithal projections of the two-layer systems comprising one 2D unit cell are plotted side by side with the corresponding perspective views. The two layers can be easily identified by the color of the atoms belonging to each of them. At every plot, the inter-planar interacting peripheral nitrogen atoms are marked with a circle and linked with a black line. A label indicates the corresponding inter-atomic distance in angstroms. At some points, a slight relative displacement of the overlapping atoms can be appreciated in the zenithal projection and this can be attributed to the small tilts featured by the melem units in the 2D polymer [9]. It is remarkable that individual $\mathrm{N}-\mathrm{N}$ distances can be smaller than the average layer separation of $3.22 \AA$ but are always larger than twice the Van der Walls radius of nitrogen, i.e., $3.1 \AA$. This shorter inter-atomic distances are also allowed for by the local oscillations of the positions of individual atoms around the average layer center due to the tilting of the melem units in the polymer sheets. The lowest energy configurations shown in Fig. 15 are characterized by the existence of a large number of peripheral $\mathrm{N}-\mathrm{N}$ inter-planar interacting sites with a small inter-atomic distance. Also, there is a very small overlap between the melem units in the two planes, indicating a negligible contribution from interactions with $\pi$-systems or between inter-planar $\pi$-systems.

Fig. 16 shows four optimized geometries with consecutive values of formation energies and in the intermediate region of the full range of energies. Again, interactions between peripheral nitrogen atoms in the 

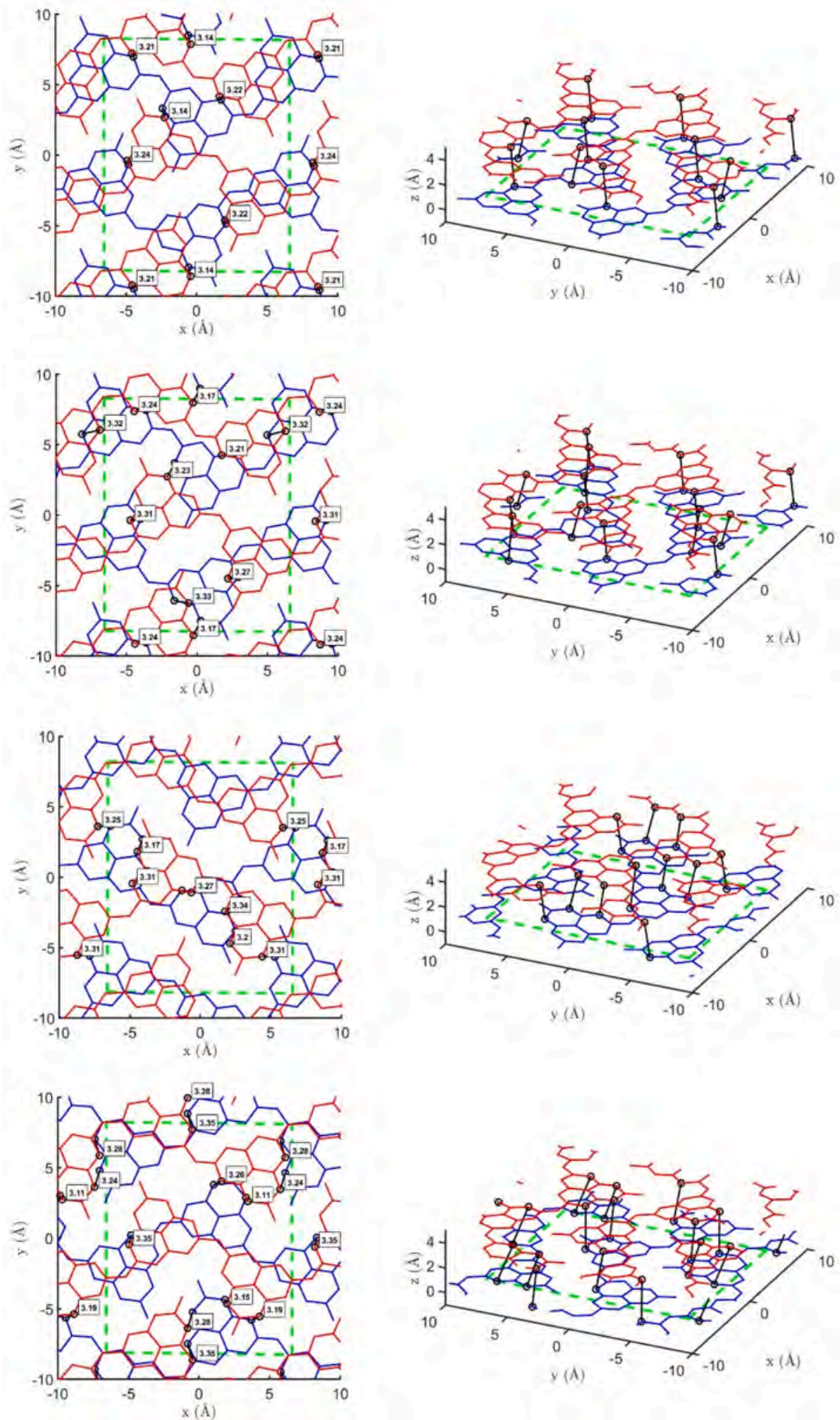

Fig. 15. The figure displays the heavier atoms ( $\mathrm{N}$ and C) lying within a unit cell for each of the four lowest energy two-layer arrangements. Atoms belonging to each melon sheet are identified by the same color where red and blue correspond, respectively, to the top and bottom sheets. The left panels show zenithal views and all the peripheral $\mathrm{N}-\mathrm{N}$ interplanar pairs with distances smaller than $3.35 \AA$ are marked with black circles and linked with a solid black trace. The inter-atomic distances (in angstroms) are also labelled. The right panels show the corresponding perspective views. The total energies of the geometries displayed are, from top to bottom, $-19,115.585960 \quad \mathrm{eV}, \quad-19,115.585380 \mathrm{eV}$, $-19,115.581080 \mathrm{eV}$, and $-19,115.579650 \mathrm{eV}$. (For interpretation of the references to color in this figure legend, the reader is referred to the web version of this article.) two layers with a distance below $3.35 \AA$ have been highlighted. These optimized geometries with a higher total energy display a lesser number of interacting peripheral nitrogen atom pairs and larger inter-atomic separations. Also, in some of the cases, the overlapping between heptazine cores in the two layers becomes larger than in the lowest energy cases, indicating the possibility of a larger contribution from interactions between $\pi$-systems. Fig. 17 plots similar results for three of the highest energy configurations optimized using the PM6 Hamiltonian. Only one of the geometries displays interactions of peripheral nitrogen atoms with inter-atomic distances below $3.35 \AA$ and, in this case, the inter-atomic distances are close to this threshold. On the other hand, these three geometries display large overlaps between the melem units in the two planes indicating a dominance of $\pi-\pi$ interactions. It can be concluded that inter-planar linkage based on $\pi-\pi$ interactions results in a 

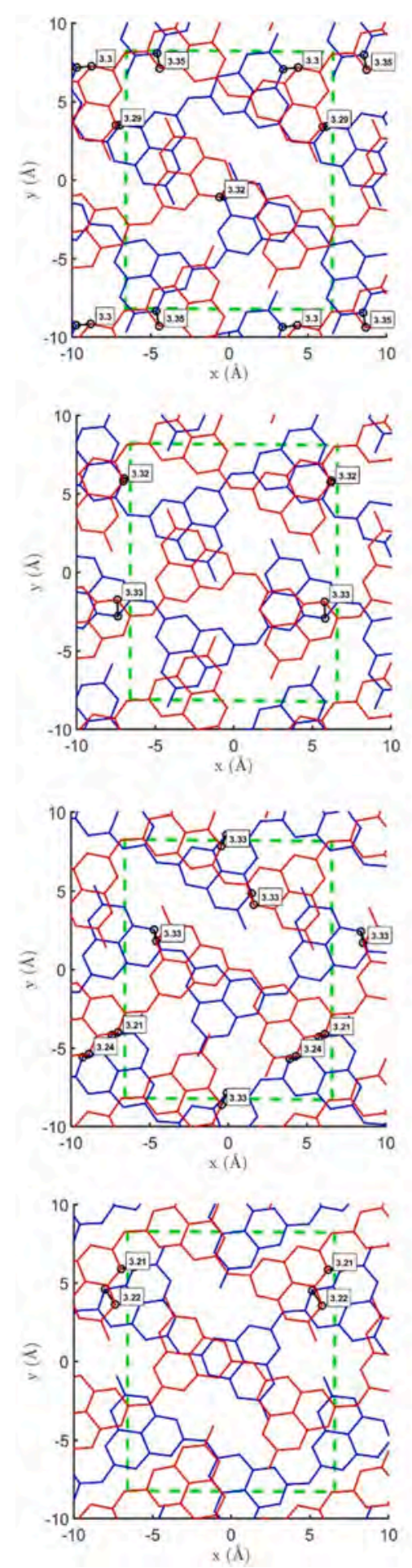
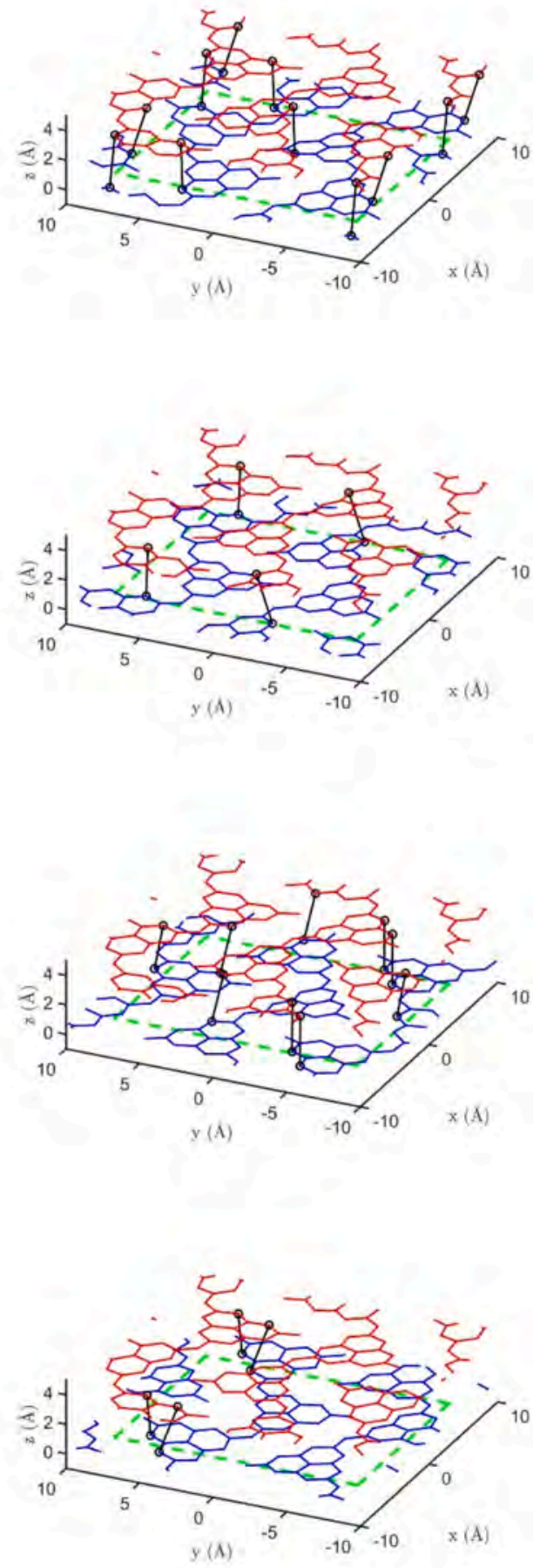

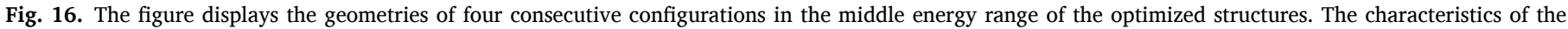

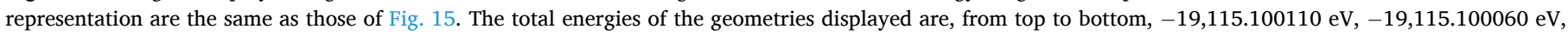
$-19,115.100010 \mathrm{eV}$, and $-19,115.099920 \mathrm{eV}$.

higher formation energy and larger inter-planar separations than in the linkage based on peripheral $\mathrm{N}-\mathrm{N}$ interactions.

\section{Discussion}

We have performed an experimental and theoretical study of a thin film sample grown on a ceramic glass substrate using thermal CVD in air atmosphere. For reference purposes, we have used the results obtained from the characterization of a formerly synthesized powder polymeric carbon nitride and we have also evaluated the deposition on an ordinary glass, with a diminished performance at the synthesis temperature of $600{ }^{\circ} \mathrm{C}$.

The FTIR measurements show that all the samples display the same vibrational modes in the whole frequency band, which indicates that the materials analyzed share the same molecular structure. Nevertheless, in the thin film sample, the absorption at the backbone vibration at 815 

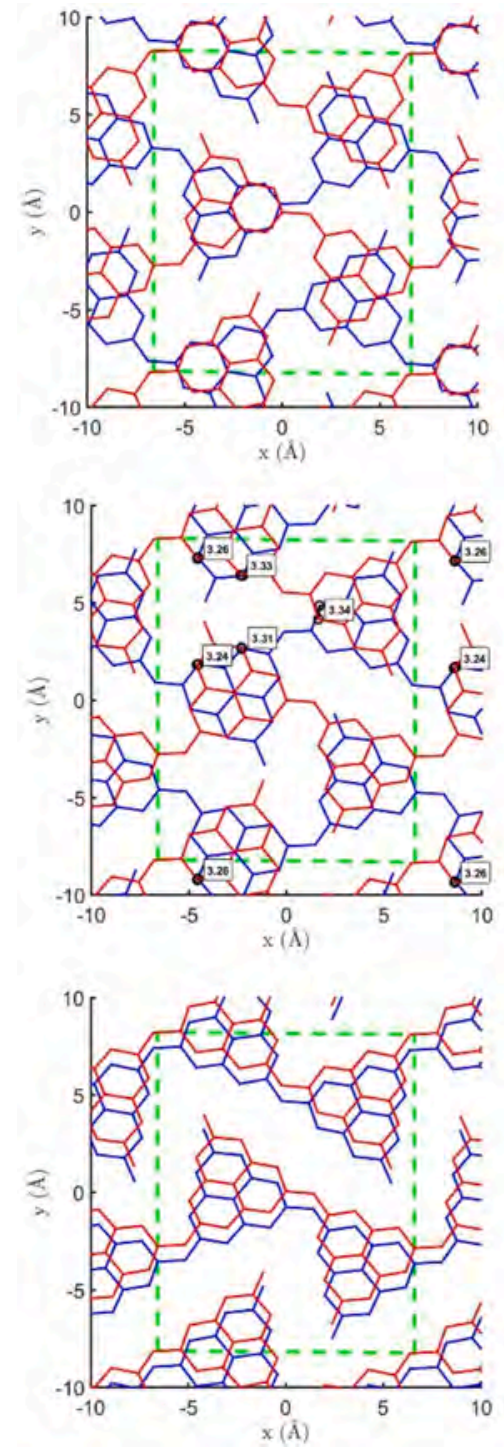
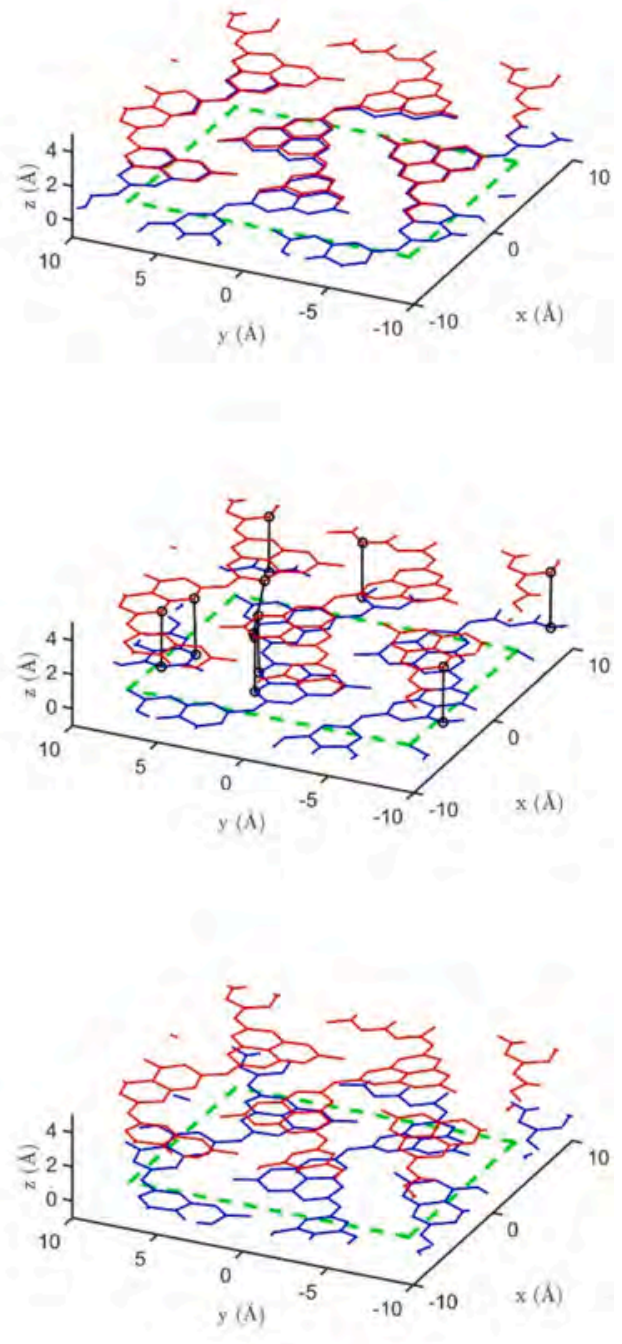

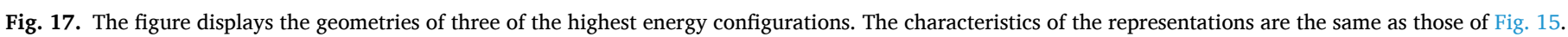
The total energies of the geometries displayed are, from top to bottom, $-19,114.163570 \mathrm{eV},-19,114.141050 \mathrm{eV}$, and $-19,114.110970 \mathrm{eV}$.

$\mathrm{cm}^{-1}$ is particularly pronounced as compared with the other vibrations, which can be attributed to an improved organization of the material layers. This interpretation is reinforced by the XRD measurements with the X-ray beam incident on the film plane, which show a very clear signal from interplanar reflections. At the same time, there is a complete absence of the type of the reflections that would be associated to a repetitive patterning in the stacking structure. Moreover, when the sample is rotated to analyze the in-plane diffraction in the SAED measurement, any trace from the $3 \mathrm{D}$ ordering in the material structure fully vanishes, therefore confirming the complete lack of crystalline ordering which is, otherwise, compatible with a very good stacking of the layers conforming the thin film. The relative shift of the Raman $G$ band is also consistent with the properties of the material described above.

In our theoretical analysis, we have obtained optimized geometries for initial conditions that systematically explore the possible conformations of a two-layer arrangement. Instead of well-defined minima, the results display a multiplicity of different configurations with similar low energy values covering broad regions in the conformation space. The existence of a multiple manifold of low energy two-layer conformations does not preclude the organization of the material in a proper crystal, especially if this is of microscopic size, and there exist experimental evidences of such crystallites in different types of samples. Nevertheless, it definitely makes the production of large size crystals extremely difficult and favors mostly amorphous syntheses with spatial conformations that will depend on the specific growth procedure.

A detailed study of the available inter-layer binding mechanisms has permitted to explain the energy ordering of the conformation space obtained in the computations. Three different types of binding forces have been identified. Hydrogen bonds keep together the melon chains in the polymer layers and are also predominantly responsible for the formation of the molecular crystals of the melem monomers [28,29]. Nevertheless, the geometry restrictions arising from the stacking of the polymer layers hinders the efficiency of these hydrogen bindings that have a negligible contribution in the formation of the thin film. Other types of possible interlayer binding mechanisms include interactions between peripheral nitrogen atoms and $\pi-\pi$ interactions. The generation of a very large set of optimized geometries covering the whole space of possible initial conditions has permitted to explore the relative efficiency of these two types of Van der Waals mechanisms. The interactions between peripheral nitrogen atoms dominate at the most energetically favorable geometries, with the smallest inter-layer separations. $\pi-\pi$ interactions, on the other hand, result in the highest energy configurations with the largest inter-layer separations. 


\section{Conclusion}

A theoretical and experimental study of the properties of a CVDsynthesized carbon nitride thin film has been performed. The comparison of the FTIR measurements with those of a formerly synthesized graphitic carbon nitride powder indicates that both materials share the same molecular composition but differ in their structural organization. The results obtained from the characterizations of the material are simultaneously consistent with a well-organized stacking of the polymer sheets in the 3D solid and a fully amorphous conformation. These apparently contradictory properties have been explained by the conclusions of an extensive computational study based on semi-empirical quantum chemistry methods.

The reported synthesis procedure of the carbon nitride thin film is based on a simple thermal CVD in air atmosphere. The characterization of the film surface and the data obtained from the XPS analysis indicate the presence of remains of the precursors placed in small islands spread on top of the thin film surface. Further investigations will include the trimming of the processing time to control the film quality.

\section{CRediT authorship contribution statement}

Pedro Chamorro-Posada: Conceptualization, Investigation, Writing - original draft. Roberto C. Dante: Conceptualization, Investigation, Writing - review \& editing. José Vázquez-Cabo: Investigation. Denisse G. Dante: Investigation. Pablo Martín-Ramos: Investigation. Óscar Rubiños-López: Investigation. Francisco M. Sánchez-Arévalo: Investigation.

\section{Declaration of competing interest}

The authors declare that they have no known competing financial interests or personal relationships that could have appeared to influence the work reported in this paper.

\section{Acknowledgement}

FIB and TEM and SEM electronic microscopy measurements, Raman, XPS and profilometry characterizations have been carried out at CACTI at Universidade de Vigo. PCP acknowledges financial support from Junta de Castilla y León, project number VA296P18.

\section{References}

[1] R.C. Dante, F.M. Sánchez-Arévalo, P. Chamorro-Posada, J. Vázquez-Cabo, L. Huerta, L. Lartundo-Rojas, J. Santoyo-Salazar, O. Solorza-Feria, A. Diaz-Barrios, T. Zoltan, F. Vargas, T. Valenzuela, F. Muñoz-Bisesti, F.J. Quiroz-Chávez, Synthesis and characterization of Cu-doped polymeric carbon nitride, Fullerenes, Nanotubes, Carbon Nanostruct. 24 (2015) 171-180.

[2] A. Naseri, M. Samadi, A. Pourjavadi, A.Z. Moshfegh, S. Ramakrishna, Graphitic carbon nitride (g-C3N4)-based photocatalysts for solar hydrogen generation: recent advances and future development directions, J. Mater. Chem. A 5 (2017) 23406-23433.

[3] T.R. Chetia, M.S. Ansari, M. Qureshi, Graphitic carbon nitride as a photovoltaic booster in quantum dot sensitized solar cells: a synergistic approach for enhanced charge separation and injection, J. Mater. Chem. A 4 (2016) 5528-5541.

[4] R. Zhang, Y. Wang, Z. Zhang, J. Cao, Highly sensitive acetone gas sensor based on g-C3N4 decorated MgFe2O4 porous microspheres composites, Sensors, 18, 2018.

[5] T. Lin, L. Zhong, J. Wang, L. Guo, H. Wu, Q. Guo, F. Fu, G. Chen, Graphite-like carbon nitrides as peroxidase mimetics and their applications to glucose detection, Biosens. Bioelectron. 59 (2014) 89-93.

[6] J. Liebig, Uber einige Stickstoff - Verbindungen, Annalen der Pharmacie 10 (1834) $1-47$.
[7] L. Pauling, J.H. Sturdivant, The structure of cyameluric acid, hydromelonic acid and related substances, Proc. Natl. Acad. Sci. 23 (1937) 615-620.

[8] B.V. Lotsch, M. Döblinger, J. Sehnert, L. Seyfarth, J. Senker, O. Oeckler, W. Schnick, Unmasking melon by a complementary approach employing electron diffraction, solid-state NMR spectroscopy, and theoretical calculations-structural characterization of a carbon nitride polymer, Chem. Eur. J. 13 (2007) 4969-4980.

[9] P. Chamorro-Posada, J. Vázquez-Cabo, F. M. Sánchez-Arévalo, P. Martín-Ramos, J. Martín-Gil, L. M. Navas-Gracia, and RobertoC.Dante, "2D to 3D transition of polymeric carbon nitride nanosheets," J. Solid State Chem. , Vol. 219, 232-241, 2014.

[10] P. Chamorro-Posada, P. Martín-Ramos, F.M. Sánchez-Arévalo, R.C. Dante, Molecular dynamics simulations of nanosheets of polymeric carbon nitride and comparison with experimental observations, Fullerenes, Nanotubes, Carbon Nanostruct. 26 (2018) 137-144.

[11] R.C. Dante, P. Martin-Ramos, L.M. Navas-Gracias, F.M. Sanchez-Arevalo, J. MartinGil, Polymeric carbon nitride nanosheets, J. Macromol. Sci. B Phys. 52 (2013) 623-631.

[12] F. Fina, S.K. Callear, G.M. Carins, J.T.S. Irvine, Structural investigation of graphitic carbon nitride via XRD and neutron diffraction, Chem. Mater. 27 (2015) 2612-2618.

[13] P. Chamorro-Posada, "A study of the therahertz spectra of crystalline materials (polyethylene, poly(vinylidene fluoride) form II, and a-D-Glucose) using NDDO semiempirical methods, Journal of Applied Spectroscopy, Vol. 85, No. 3, July, 2018.

[14] J.D.C. Maia, G.A. Urquiza Carvalho, C.P. Mangueira, S.R. Santana, L.A.F. Cabral, G. B. Rocha, J. Chem. Theory Comput. 8 (2012) 3072-3081.

[15] R.C. Dante, P. Martín-Ramos, F.M. Sánchez-Arévalo, L. Huerta, M. Bizarro, L. M. Navas-Gracia, J. Martín-Gil, Synthesis of crumpled nanosheets of polymeric carbon nitride from melamine cyanurate, J. Solid State Chem. 201 (2013) 153-163.

[16] R.C. Dante, P. Martín-Ramos, L.M. Navas-Gracia, F.M. Sánchez-Arévalo, J. MartínGil, Polymeric carbon nitride nanosheets, J. Macromol. Sci., Phys., 52 (2013) 623-631.

[17] R.C. Dante, F.M. Sánchez-Arévalo, P. Chamorro-Posada, J. Vázquez-Cabo, L. Huerta, L. Lartundo-Rojas, J. Santoyo-Salazar, O. Solorza-Feria, Supramolecular intermediates in the synthesis of polymeric carbon nitride from melamine cyanurate, J. Solid State Chem. 226 (2015) 170-178.

[18] J.J.P. Stewart, Optimization of parameters for semiempirical methods V: modification of NDDO approximations and application to 70 elements, J. Mol. Mod. 13 (2007) 1173-1213.

[19] J. Ridley, M. Zerner, An intermediate neglect of differential overlap technique for spectroscopy: pyrrole and the azines, Theor. Chim. Acta 32 (1973) 111.

[20] M.C. Zerner, G.H. Loew, R.F. Kirchner, U.T. Mueller-Westerhoff, An intermediate neglect of differential overlap technique for spectroscopy of transition-metal complexes. Ferrocene, J. Am. Chem. Soc. 102 (1980) 589.

[21] F. Neese, "The ORCA program system," Wiley Interdiscip. Rev.: Comput. Mol. Sci. 2, 73 (2012)

[22] A.C. Ferrari, S.E. Rodil, J. Robertson, Interpretation of infrared and Raman spectra of amorphous carbon nitrides, Phys. Rev. B 67 (2005) 155306.

[23] J. Yang, X. Gong, G. Wang, Structure, aromaticity, stability, and energetic performance of the analogues and derivatives of s-heptazine, J. Mol. Model. 20 (2014) 2379.

[24] R. Nozawa, J. Kim, J. Oh, A. Lamping, Y. Wang, S. Shimizu, I. Hisaki, T. Kowalczyk, H. Fliegl, D. Kim, H. Shinokubo, Three-dimensional aromaticity in an antiaromatic cyclophane, Nat. Commun. 10 (2019) 3576.

[25] H-K. Jeong, Y. P. Lee, R.J.W.E. Lahaye, M-H. Park, K.H. An, I.J. Kim, C-W. Yang, C. Y. Park, R.S. Ruoff, and Y.H. Lee, "Evidence of graphitic AB stacking order of graphite oxides,” J. Am. Chem. Soc. 2008, 130, 1362-1366 (2008)

[26] L. Houben, A.N. Enyashin, Y. Feldman, R. Rosentsveig, D.G. Stroppa, M. Bar-Sadan, Diffraction from disordered stacking sequences in MoS2 and WS2 fullerenes and nanotubes, J. Phys. Chem. C 116 (2012) 24350-24357.

[27] P. Chamorro-Posada, J. Vázquez-Cabo, O. Rubiños-López, J. Martín-Gil, S. Hernández-Navarro, P. Martín-Ramos, F. M. Sánchez-Arévalo, A. V. Tamashausky, C. Merino-Sánchez, R. C. Dante, "THz TDS study of several sp2 carbon materials: graphite, needle coke and graphene oxides," Carbon 98, 484-490 (2106).

[28] B. Jürgens, E. Irran, J. Senker, P. Kroll, H. Müller, W. Schnick, Melem (2,5,8triamino-tri-s-triazine), an important intermediate during condensation of melamine rings to graphitic carbon nitride: synthesis, structure determination by X-ray powder diffractometry, solid-state NMR, and theoretical studies, J. Am. Chem. Soc. 125 (2003) 10288-10300.

[29] A. Sattler, W. Schnick, On the crystal structure of melem $\mathrm{C}_{6} \mathrm{~N}_{7}\left(\mathrm{NH}_{2}\right)_{3}$, Z. Anorg. Allg. Chem. 632 (2006) 238-242.

[30] A. Zambon, J.-M. Mouesca, C. Gheorghiu, P. A. Bayle, J. P'ecaut, M. Claeys-Bruno, S. Gambarelli and L. Dubois, "s-Heptazine oligomers: promising structural models for graphitic carbon nitride ," Chem. Sci. 7, 945-950 (2016). 